
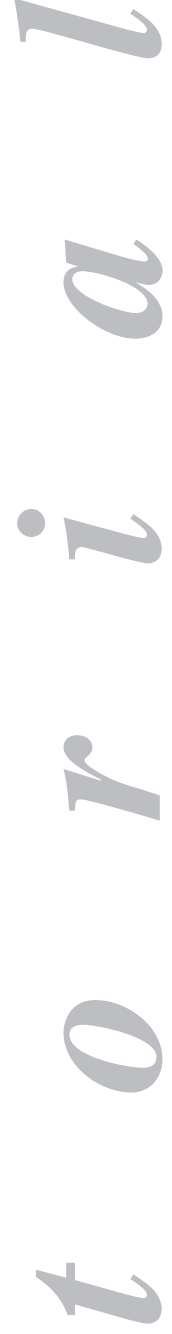

P
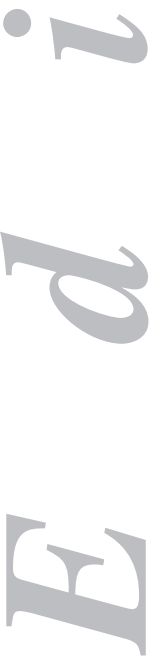

Intestinal transplantation

Until nearly three decades ago the small bowel was considered an organ unsuitable for transplantation, since its high lymphoid tissue contents, strong expression of histocompatibility antigens, and colonization by microorganisms made rejection and infection the rule. With the introduction of tacrolimus (FK-506) in 1989 the risk for rejection and fatal infection decreased, and the procedure became a reality and was initially used to rescue patients with intestinal failure. For almost a decade, however, complex patient care and the need for strong, long-term immune suppression prevented its widespread use. During the last few years technical innovations, new immunosuppressing protocols, and improved postoperative management have significantly increased the efficacy of this therapeutic procedure, which is more commonly used for patients with intestinal failure. Thus, data reported by the International Intestinal Transplant Registry in 2003 reflect an increased number of bowel transplants, particularly from 1997 on, as well as improved patient and graft survival (1).

Intestinal transplantation is therefore a feasible therapeutic option for patients suffering from intestinal failure. This term is applied when patients cannot obtain adequate nutrition from an enteric diet, and thus need a portion of their caloric requirements from parenteral nutrition (PN) (2). Most commonly, intestinal failure results from surgical resection in order to treat the patient's condition, which yields a short bowel; in other instances, the bowel is sufficiently long, but unable to absorb fluids and nutrients necessary to meet the body's metabolic demands, thus resulting in intestinal failure. Causes differ in adults and children, but a short bowel is the most common indication (1).

Thus in pediatric patients, the most common causes of intestinal failure requiring an intestinal transplant include gastroschisis (21\%), volvulus (17\%), dismotility $(16 \%)$, necrotizing enterocolitis $(12 \%)$, intestinal atresia $(8 \%)$, and microvillous inclusion disease (6\%). In contrast, in adults, ischemia (23\%), Crohn's disease (14\%), gastrointestinal neoplasms (16\%), and trauma (10\%) represent the most common indications leading to intestinal transplantation. Also a retransplant was performed in $8 \%$ of pediatric recipients and $6 \%$ of adult recipients (3).

Two studies have shown that patient survival at 1 year after intestinal transplant is 92 and $88 \%$, similar to survival with PN $(4,5)$. However, while results have improved over time, long-term survival is not as good as with PN. Overall survival rates for patients with home parenteral nutrition is $85 \%$ at 3 years (6), while 3 -year survival is $61 \%$ with intestinal transplantation (7).

Thus, the indication for intestinal transplantation is currently restricted to patients where parenteral nutrition reached its limits, either from administration difficulties or because of serious complications contraindicating it. In 2003, the American Gastroenterological Association (AGA) (8) proposed intestinal transplant as a therapeutic option for patients with intestinal failure and life-threatening complications, either attributed 
to intestinal failure and/or derived from parenteral nutrition. Specifically, criteria for transplantation included: a) liver involvement (elevated bilirubin and/or liver enzymes, splenomegaly, thrombopenia, esophagogastric varices, coagulopathy, stoma bleeding, liver fibrosis or cirrhosis); b) thrombosis in two major venous accesses (subclavia, jugular, or femoral vein); c) sepsis in relation to a central access (2 episodes a year, or 1 fungemia episode, or septic shock, or respiratory distress syndrome); and d) frequent severe dehydration events. In summary, the two most common indications included $\mathrm{PN}$-associated liver disease and a progressive loss of venous accesses for continued PN (9). Nevertheless, as intestinal transplantation advances other factors such as quality of life may be potential indications in selected cases (10). Hence, the following should be considered eligible for intestinal transplant -tumors with local abdominal invasion (desmoid tumor), non-reconstructable gastrointestinal tract, primary loss of an intestinal graft, and poor quality of life secondary to intestinal failure. However, since PN is usually well tolerated by most patients, the indication for intestinal transplantation should be carefully considered within the context of each individual patient, and riskbenefit ratios should be assessed.

Three distinct types of intestinal transplant exist: intestine alone, hepatointestinal (intestine and liver), and multivisceral, which includes the stomach, duodenum, pancreas, intestine and liver, and sometimes other organs such as the kidneys (1).

Hepatointestinal transplant is indicated for patients with PN-related liver disease without intestinal adjustment or irreversible intestinal damage. It would not be indicated for reversible liver dysfunction. However, it is indicated for liver disease progression as suggested by laboratory parameters (hyperbilirubinemia, hypertransaminasemia, hypoalbuminemia, coagulopathy) or evidence of cirrhosis (fibrosis in liver biopsy) and portal hypertension (hepato-splenomegaly, ascites, esophageal varices) as a sign of irreversible liver involvement. Dual hepatointestinal transplantation is also indicated for patients with mesenteric vascular thrombosis as a result of protein $\mathrm{S}$ or $\mathrm{C}$ deficiency when associated complications develop despite anticoagulation therapy and PN. It should be highlighted that a small group of -mainly pediatric- patients with PNdependent intestinal failure develop a rapidly progressing liver dysfunction before intestinal adjustment completion. For such cases a liver transplant with no intestinal transplant would be initially indicated since intestinal adjustment after liver transplant may render PN unnecessary for many of these children (11).

$\mathrm{PN}$-associated liver disease must be recognized as a condition quite different to liver disease in patients with a normal intestine. Patients with a short bowel, most of those with intestinal failure, have a low mesenteric flow and fewer porto-systemic shunts. Hence they rarely develop esophageal varices, clinically relevant ascites, or spontaneous bacterial peritonitis. However, hyperbilirubinemia and hypersplenismderived effects, particularly splenomegaly and thrombopenia, represent the primary evidence of liver disease in these patients. In contrast with other liver conditions, patients with intestinal and liver failure are rarely malnourished as they receive PN, and liver encephalopathy and coagulopathy are relatively late findings. It must be pointed out that small children with a short bowel are more likely to develop liver disease even earlier and more progressively than older children and adults. It is therefore more frequent that a high proportion of intestinal transplants are combined liver-bowel procedures in children (9).

On the other hand, patients with hepatointestinal transplantation have a shorter survival when compared to intestinal transplantation alone, albeit with a lower incidence of rejection; furthermore, such episodes are even milder. The liver-induced immunotolerance mechanism is as yet unknown (12). 
Multivisceral transplantation may be indicated following a gastrointstinal tract excision for an all-invading tumor involving most intraabdominal organs or when motility disorders involve the gastrointestinal tract in all its length. It is not indicated for patients with malignancies; however, patients with benign tumors (particularly desmoid tumors) may have extensive intraabdominal involvement and be eligible. Such decisions should be made on an individual basis as the surgical technique may be complex and require careful presurgical planning (9).

A transplant's main goal is to increase survival for patients with intestinal failure and life-threatening complications, as well as to prevent immune-mediated graft loss, to maximize graft survival as much as possible, and to minimize long-term morbidity (13).

One-year survival for patients transplanted later than 1988 is $77 \%$, and graft survival is $65 \%$ for intestine-only transplants, 60 and $59 \%$ for hepatointestinal transplants, and 66 and $61 \%$ for multivisceral transplants, respectively (3). Survival is higher for intestine versus liver plus intestine transplants -in the former case, besides a milder disease, grafts may be removed should a serious complication arise, and parenteral nutrition may be resumed (9). According to data from the International Intestinal Transplant Registry (14), 484 intestinal transplant recipients were alive by May 2003 (52\%). Most common causes of mortality included sepsis (49\%), rejection $(11 \%)$, technical complications $(9 \%)$, lymphoma $(6 \%)$, and respiratory failure $(6 \%)$. When analyzing factors influencing survival the following were detected: learning curve in sites (better when more than 10 had been performed), patient wait for transplantation in their homes or in the hospital, patient age, induction therapy, and pretreatment (14). Upon their analysis of risk factors, the Pittsburgh team -who performed one third of all intestine transplants worldwide- identified that high-potency immune suppression was the main risk factor for survival, while other factors included: prolonged duration of graft cold ischemia, number of previous surgeries, prolonged surgery, inclusion of a colonic segment within the graft, and incidence of lymphoproliferative disease and CMV (3).

Postsurgical care for the intestinal transplant recipient are complex and require a specialized, multidisciplinary team (15). Their key role must be: immune suppressing therapy for rejection prevention; infection prevention and treatment; monitoring potential immunoproliferative disease, graft versus host disease, nutritional support, and fluid and electrolyte management.

The introduction of novel immune suppressing protocols has changed the course of intestinal transplant. Immune suppression is the most debated aspect of posttransplant management. Many therapeutic regimens have been described. All of them are empirical, and while different, they all attempt to reduce rejection rates while concomitantly limiting toxicity as much as possible. Tacrolimus is the drug of choice for immune suppression in intestinal transplantation (13). Furthermore, induction using either antibodies against the interleukin-2 receptor (basiliximab, daclizumab), campath-1H (alemtuzumab), or thymoglobuline is also used. Until recently corticoids were also used, but there is currently a trend to use tacrolimus with no steroids, as is the case with Miami and Pittsburgh teams for adult intestinal transplantation with good results (overall patient survival at 1 year is $92 \%$, with a graft survival rate of $89 \%$ after one year) (1).

An early diagnosis of rejection is a most important aspect of postoperative care. Early clinical manifestations (impaired debit from colostomy, pain, fever,...) and histological criteria (16) are definitive evidence for rejection diagnosis and monitoring. Endoscopic surveillance with multiple mucosal biopsies should occur once or 
twice a week during the immediate postoperative period. However the frequency of routine endoscopies varies considerably among centers (13). As the ileum is more susceptible to rejection versus the jejunum, endoscopies are performed through a temporary funnel or simple ileostomy. Upper endoscopy and/or enteroscopy is only required for patients with upper gastrointestinal symptoms, clinically suspected rejection with normal ileal biopsy, or other specific indications (1). Zoom video endoscopy or serum citruline levels may help diagnose early rejection (3). All sites initially manage rejection using steroid boluses and increasing immune suppression with tacrolimus; the use of anti-lymphocyte antibodies (OKT3, thymoglobuline) is restricted to corticoid-unresponsive severe rejection (13).

Another risk of intestinal transplantation is infection, which still represents a major cause of morbidity and mortality. In an Italian series of 19 patients with intestinal transplant $94 \%$ developed at least one bacterial infection event, $67 \%$ had viral infection, and $28 \%$ had fungal infection (17). Risks for bacterial and fungal infection are reduced with antibiotic prophylaxis during the immediate postoperative period, systematic blood cultures, and early central access withdrawal. Furthermore, the risk for infection by CMV and Epstein-Barr virus is linked to lymphoma development post-transplant. Hence most centers use long-term antiviral therapy with gancyclovir with or without hyperimmune globuline against CMV, as well as monitoring to routinely detect viruses within the blood and tissues by measuring CMV antigenemia and using PCR for VEB $(3,13)$.

Another goal of intestinal transplantation is to attain enteral autonomy for patients. Most recipients meet all their nutritional requirements enterally, and may discontinue PN within 4-6 weeks post-transplant, which favors graft adjustment, simplifies postoperative care, and clears potential PN-related risks, particularly infection and central access thrombosis (1). Bowel graft absorptive capabilities are usually influenced by both immune and non-immune events, including severe graft preservation damage, rejection, viral enteritis, systemic infection, and technical failure at surgery; hence they may on occasion require a second, temporary course of PN.

Most centers initially use enteral formulas that are subsequently modified. All of them advice against refined sugar-containing food and beverages, including fruit and fruit juice, in order to prevent osmotic diarrhea (13). Thus, $81 \%$ of patients surviving the procedure achieve full gastrointestinal autonomy and are free from parenteral nutrition (18).

The quality of life of transplanted patients is an issue to consider; even in the absence of controlled studies, some authors report complete psychic and psychiatric rehabilitation in $85 \%$ of recipients surviving longer than 6 months (19). Interestingly, in Pittsburgh those surviving longer than 6 months achieve total independence for daily activities and full occupational rehabilitation in $92 \%$ of cases (1). Preliminary studies show that quality of life is seemingly good or normal -even better than when on PN- in patients with intestinal transplant (19).

Data from the International Intestinal Transplant Registry show that more than half of recipients are children at the time of transplantation, which explains why pediatric series reported $(12,20,21)$ are greater than adult series $(12,22,23)$. A pediatric intestinal transplant program is in force in our country since 1997 (20). While pediatric intestinal transplants had already been previously performed, it was not until 2002 that the first intestinal transplant for an adult patient was carried out at Hospital Ramón y Cajal (E. de Vicente, Y. Quijano, et al.) -a female with congenital familial polyposis and parenteral nutrition-associated complications (6). The one series of adult patients receiving an intestinal transplant in our country is that reported 
by Meneu et al. (24). On comparison between the pediatric and adult series we may see -as reported in the literature- a higher rate of hepatointestinal transplants in children as compared to adults, as well as a longer waiting time because of fewer small-organ donors (9).

The series by Meneu et al. (24), while short because of the program's recent start with a non-standard procedure, shows good short-term results. As with other solid organ grafts, patient short- and long-term survival will improve over time; should quality of life turn out good, intestinal transplantaion will be offered to all patients with intestinal failure before the development of PN-derived complications.

\section{Garfia Castillo}

Service of Digestive Diseases. Hospital Universitario "12 de Octubre”. Madrid, Spain

\section{REFERENCES}

1. Abu-Elmagd KM. Intestinal transplantation for short bowel syndrome and gastrointestinal failure: current consensus, rewarding outcomes, and practical guidelines. Gastroenterology 2006; 130 (2 Supl. 1): S132-7.

2. O’Keefe SJ, Buchman AL, Fishbein TM, Jeejeebhoy KN, Jeppsen PB, Shaffer J. Short bowel syndrome and intestinal failure: Consensus definitions and overview. Clin Gastroenterol Hepatol 2006; 4: 6-10.

3. Flynn B, Park K, Bond G, McGhee W, Mazariegos G, Sindhi R, et al. Immunosuppressant strategies for intestinal transplantation: a review of a tolerogenic regimen. Prog Transplant 2005; 15: 60-4.

4. Sudan DL, Kaufman SS, Shaw BW Jr. Isolated intestinal transplantation for intestinal failure. Am J Gastroenterol 2000; 95: 1506-15.

5. Fishbein TM, Kaufman SS, Florman SS. Isolated intestinal transplantation: Proof of clinical efficacy. Transplantation 2003; 76: 636-40.

6. Gajate Martín L, Elías Martín E, Martínez Pérez A, Carrasco Sedal C, Álvarez Utrera F, Pablo de Pajares A, et al. Trasplante intestinal del adulto. Situación actual y perspectivas de futuro. Rev Esp Anestesiol Reanim 2004; 51: 537-48.

7. Shiffman ML, Saabb S, Fenge S, Abecassisd MI, Tzakise AG, Goodrichf NP, et al. Liver and intestine transplantation in the United States, 1995-2004. Am J Transplantation 2006; 6: 1170-87.

8. Buchman AL, Scolapio J, Fryer J. AGA technical review on short bowel síndrome and intestinal transplantation. Gastroenterology 2003; 124: 1111-34.

9. Florman S, Kaufman SS, Fishbein T. Decision making in intestinal transplantation. Prog Transplant 2005; 15: $65-8$.

10. Robinson JI, Spencer RW. Intestinal transplantation: the evaluation process. Prog Transplant 2005; 15 : $45-53$.

11. Muiesan P, Dhawan A, Novelli M, Mieli Vergani G, Rela M, Heaton ND. Isolated liver transplant and sequential small bowel transplantation for intestinal failure and related liver disease in children. Transplantation 2000; 69: 2323-6.

12. Goulet O, Sauvat F, Ruemmele F, Caldari D, Damotte D, Cezard JP, et al. Results of the Paris program: Ten years of pediatric intestinal transplantation. Transplantation Proc 2005; 37: 1667-70.

13. Horslen S. Optimal management of the post-intestinal transplant patient. Gastroenterology $2006 ; 130$ (2 Supl. 1): S132-7.

14. Registro Internacional de Trasplante Internacional. Disponible en: www.intestinaltransplantregistry.org/

15. Samela K, Fennelly E, Brosnan M, Robinson J. Interdisciplinary approach to the management of intestinal transplant recipients: evaluation, discharge, and lifetime management. Prog Transplant 2005; 15: 54-9.

16. Ruiz P, Bagni A, Brown R, Cortina G, Harpaz N, Magid MS, et al. Histological criteria for the identification of acute cellular rejection in human small bowel allografts: Results of the pathology workshop at the VIII International Small Bowel Transplant Symposium. Transplant Proc 2004; 36: 335-7.

17. Guaraldi G, Cocchi S, Codeluppi M, Di Benedetto F, De Ruvo N, Masetti M, et al. Outcome, incidence, and timing of infectious complications in small bowel and multivisceral organ transplantation patients. Transplantation 2005; 80: 1742-8.

18. Grant D, Abu-Elmagd K, Reyes J, Tzakis A, Langnas A, Fishbein T, et al. 2003 report of the intestine transplant registry: A new era has dawned. Ann Surg 2005; 241: 607-13.

19. Sudan D. Cost and quality of life after intestinal transplantation. Gastroenterology 2006; 130 (2 Supl. 1): S158-62.

20. Hernández F, López Santamaría M, Gámez M, Murcia J, Leal N, Prieto G, et al. Resultados de un programa de trasplante intestinal. Cinco años después. Cir Pediatr 2004; 17: 145-8.

21. Kato T, Tzakis AG, Selvaggi G, Gaynor JJ, David AI, Bussotti A, et al. Intestinal and multivisceral transplantation in children. Ann Surg 2006; 243: 756-64. 
22. Lauro A, Di Benedetto F, Masetti M, Cautero N, Ercolari G, Vivarelli M, et al. Twenty-seven consecutive intestinal and multivisceral transplants in adult patients: a 4 year clinical experience. Transplant Proc 2005; 37: 2679-81.

23. Dijkstra G, Rings EH, Bijleveld CM, Van Dullemen HM, Hofker HS, Porte RJ, et al. Intestinal transplantation in The Netherlands: First experience and future perspectives. Scand J Gastroenterol Suppl 2006; 243: 39-45.

24. Meneu Díaz JC, Moreno González E, García García JI, Moreno A, Pérez B, Abradelos de Usera M, et al. Primera serie nacional de trasplante de intestino en receptores adultos. Rev Esp Enferm Dig 2006; 98 (10): 723-39. 